\title{
Encrypted and Hiding Information in Digital Images using Wavelet Transformation and LSB Technology
}

\author{
Ahmed Hamed Saleh Al-Badrani \\ Technical College of Management \\ Technical Institute, Mosul, Iraq
}

Received on: 17/09/2012

Accepted on: 30/01/2013

\begin{abstract}
Hiding information is an effective solution for the protection of copyright and confidentiality to allow a person to send the data in the middle of the cover image to a person without knowing any third party in this transmission, methods of delivering secret messages are very important. This research provides a way to hide data (which is a text file) after is encrypted adoption method (Keyword Mixed Transposition) to produce cipher text is included in Low-High coefficient wavelet transform and get a good quality image and the possibility of recovering fully embedded message and decoded without relying on the original image. Results have applied to the digital images to get inline images to the data with a high correlation coefficient when compared with the original images in addition to that they gave a few differences when calculating measurements(SNR, PSNR, MSE).
\end{abstract}

Keywords: Encrypted, hiding information, wavelet transformation, and LSB Technology

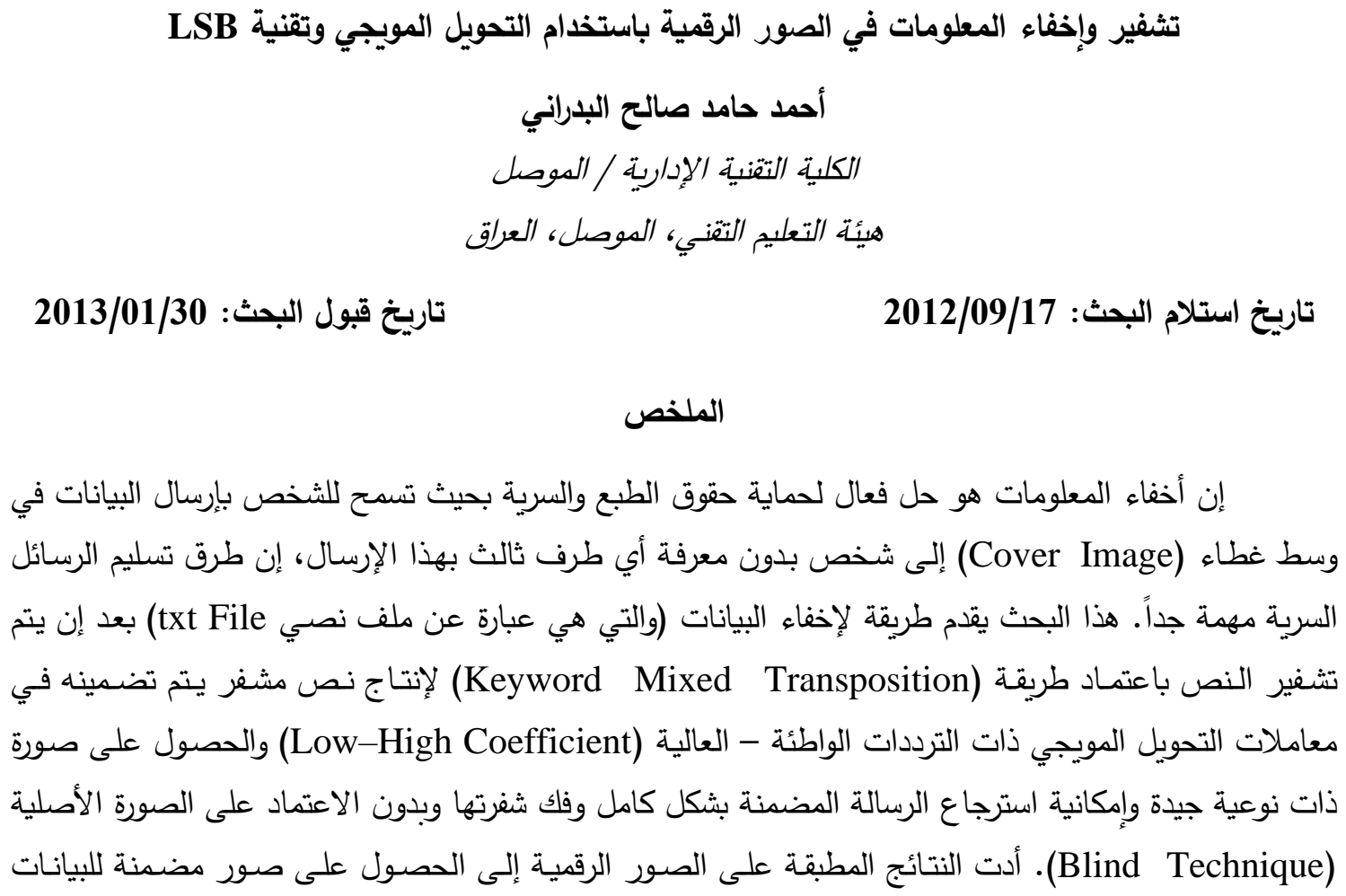


(Correlation Coefficient) ذالي عند مقارنتها مـع الصور الأصلية (Stego image) بالإضافة إلى أنها أعطت فروقات قليلة عند حساب القياسات (SNR, PSNR, MSE).

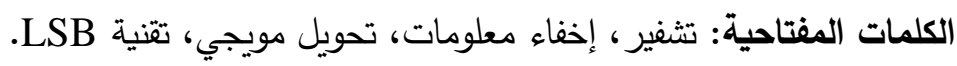

1- المقدمة

ازداد استخدام إخفاء البيانات في السنوات الأخيرة نتيجة للتطور الحاصل في الإنترنت وتقنيات معالجة

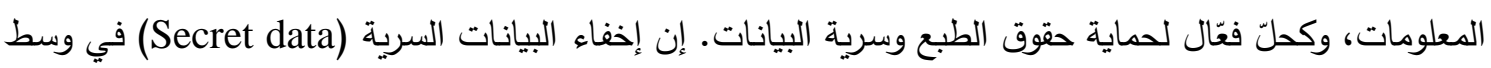

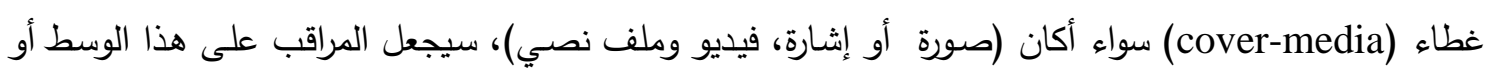
المتطفل لا يعرف بوجود رسالة مخفية في هذا الوسط، إذ إن البيانات تضمن في الصورة (تكون محمولة في بيانات

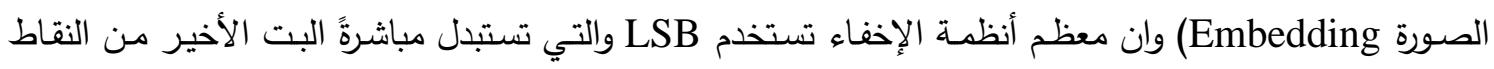
(Least Significant Bit (LSB))

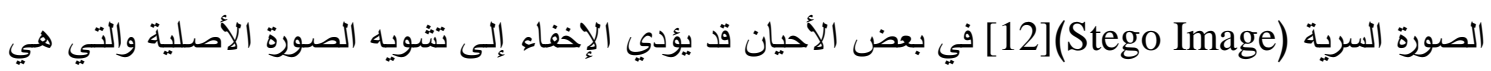

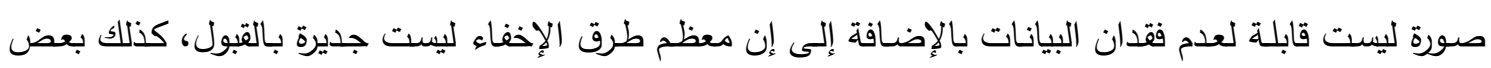

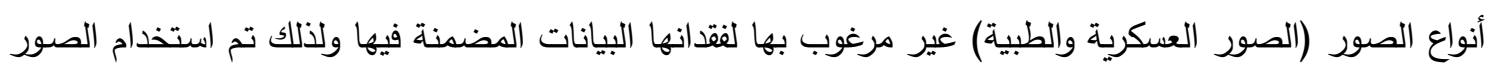
الرقمية، إذ إن نوعية(quality) الصورة المستعملة للإخفاء يجب أن تعطي صورة واضحة المعالم وفي نفس الوقت

يجب إن تسترجع الصورة الأصلية بعد انتزاع الرسالة المضمنة والحصول على كامل الرسالة المضمنة أيضاً [6].

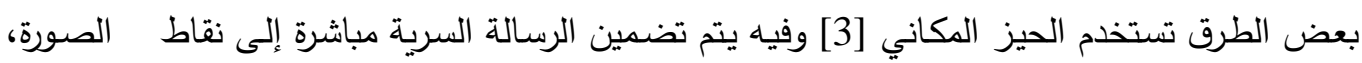
ومن هذه الطرق (الأكثر شيوعاً) Histogram-Based و(LSB)؛ والتي تعطي أعلى قدرة (High-Capacity) واقل تثوه (Distortion) كخوارزميات (Difference Expansion (DE) إذ اقترح Ni وآخرون [9] استخدام طريقة المدرج التكراري للإخفاء الذي استعمل الصفر والنقاط الضعيفة (Peak Point) للمدرج التكراري لصورة

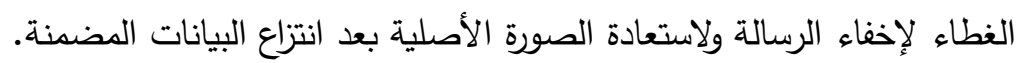
إما الطرق الأخرى فتستخدم الحيز الترددي ومن أكثر الطرق حالياً والمستخدمة لإخفاء البيانات كالتحويل المويجي وتحويل Discrete Cosine Transform) DCT Curvelet) والتي توفر قابلية عالية على المحافظة

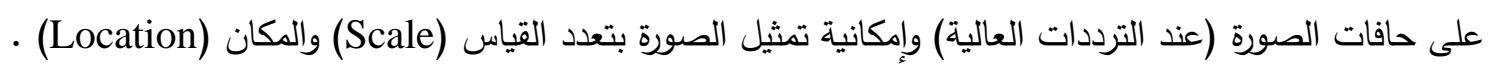

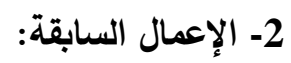

يعد موضوع إخفاء المعلومات وتثفيرها من المواضيع التي تم العمل بها مبكراً وطورت بشكل سريع

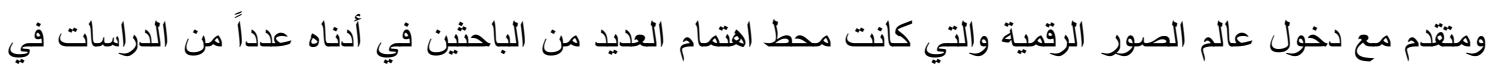
هذا المجال:

••اقترح الباحث Eman (2008) طريقة لإخفاء البيانات في الصور الثنائية، إذ يتم أولا تحديد النقاط الضوئية

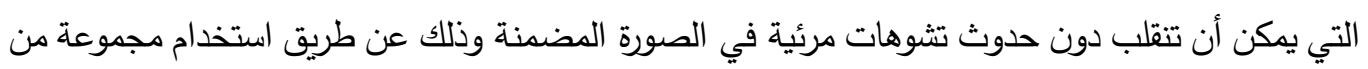

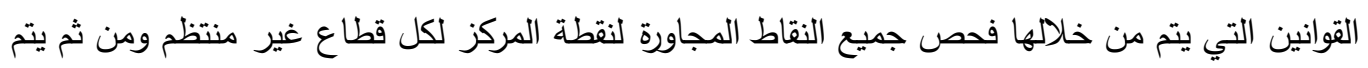
تغيير النقطة المركزية فقط في حالة مطابقة القطاع لهذه الثروط وهذه الخاصية تسمح باكتشاف البيانات المضمنة دون الرجوع إلى الصورة الأصلية، وقد أظهرت التجارب نتائج مختلفة لصور ثنائية مختلفة. 
وقدم الباحث A.A. Abdul Latef (2011) طريقة للإخفاء في الصور الملونة عن طريق تقسيمها إلى أربع أجزاء متساوية كل جزء متكون من ثلاثة قنوات (Red, Green, Blue) يتم اختيار احد هذه القنوات لكل جزء بالاعتماد على نسبة اللون العالية في ذلك الجزء، بعدها يتم تطبيق التحويل المويجي على الته الجزء

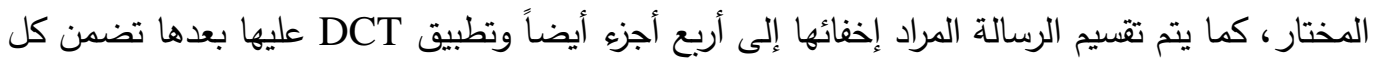
جزء دنها في الترددات العالية للتحويل المويجي لأحد أجزاء صور الغطاء للحصول على الهي الصورة السرية. أما الباحث Yong (2011) اقترح مخطط لإخفاء البيانات السرية داخل الصورة باستعمال تحويل curvelet العالية للتحويل curvelet لتضمين البيانات. • قام الباحث Chin (2005) باقتراح طريقة لإخفاء صورة بتغيير معاملات التحويل لصورة الغطاء (مثلاً (DCT)، إذ يتم إبدال كل بت من الصورة المراد تضمينها ببت من احد معاملات التحويل، إذ تبين

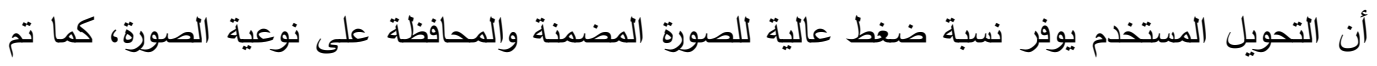
استخدم خوارزمية التشفير DES للبيانات المراد تضمينها قبل عملية التضمين لتوفير سرية.

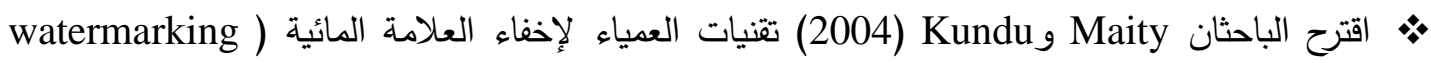
(blind المستويات للصور الملونة (multilevel wavelet LL)، إذ تم تضمين العلامة في حزمة اللون الأحمر فئر للمعاملات الترددية الواطئة. • • اقترح الباحثان Abdelwahab و Hassan (2008) استعمل المستوى الأول من التحويلات المويجية في إخفاء البيانات وتضمينها ولكن كانت البيانات المنتزعة ليست مماثلة كليا إلى النسخة المضدّة. 3- التحويلات المويجية ثنائية الإبعاد:

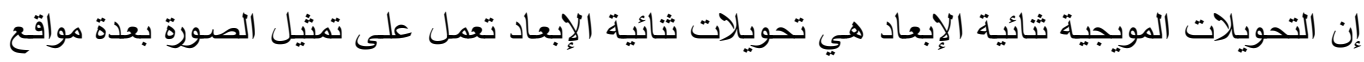

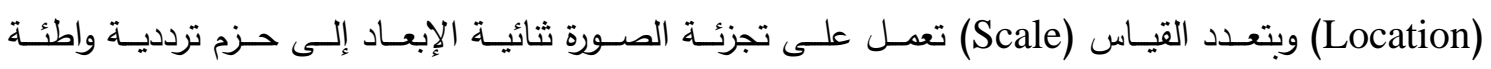

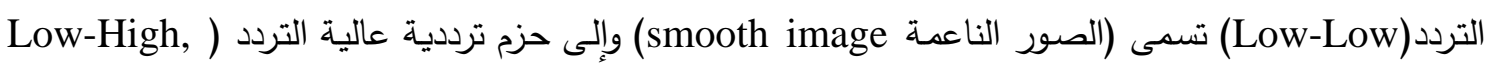
(High-Low, High-High تسمى (الصور التفصيلية detailed image). أي تعمل على تقسيم الصورة إلى تصلية أربع صور LL1, LH1, HL1, HH1 والتي تمثل المستوى الأول، وللحصول على المستوى الثاني سيتم استخدام الصورة LL1 للحصول على أربع صور أخرى هي LL2, LH2, HL2, HH2 وهكذا لبقية المستويات وكما في تلي لئي الشكل (1).017) التحويل المويجي يقدّم مهام واسعة في معالجة الصورة، إذ أن معاملاته تعطي درجة عالية من الترابط

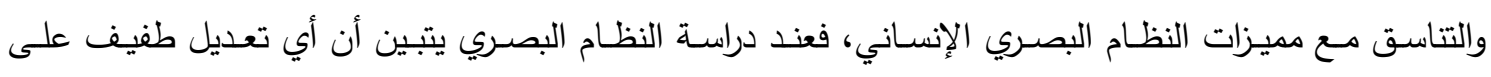
الترددات العالية من الصعب رويته بالعين البشرية [11]. لذلك سنستخدم في هذا البحث الترددات الواطئة - العالية في تضمين الرسالة السرية. 


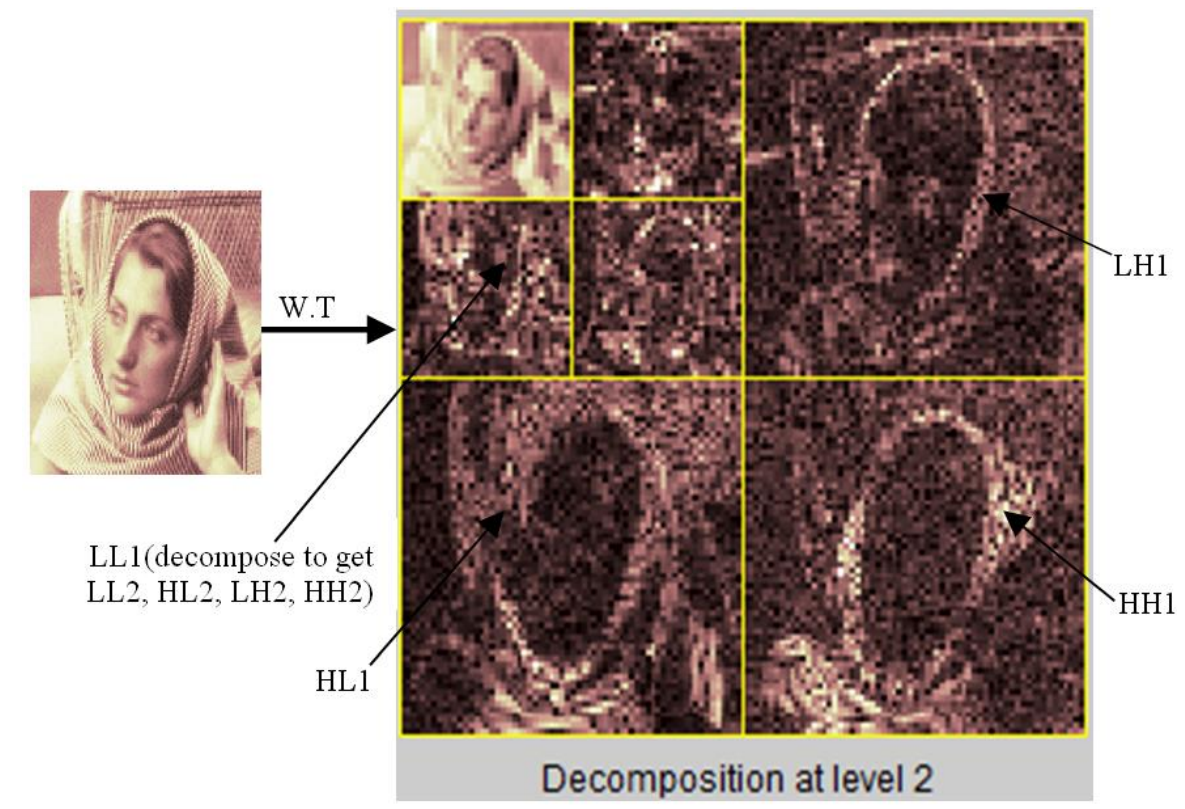

الثكل (1). التحويل المويجي لصورة Barbara لمستويين

Keyword Mixed Transposition التشفير بطريقة

تم اعتماد الطرق التعويضية كإحدى الطرق المعتمدة لتثفير النصوص والتي تعمل على إبدال النص بنص أخر ومنها طريقة خلط الأبجدية (mixed alphabet) التي تولد خليط من الحروف الأبدية بالاعتماد على تعلى مفتاح أبجدي (alphabet)عشوائي يتم اختياره لغرض تثفير الرسالة إذ تشفر النص (المسمى (plain text)

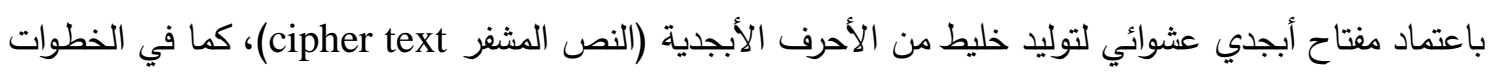
التالية: 1- إخـال المفتاح والذي هو عبارة عن كلمة عشوائية بدون تكرار للحروف (مثلا إدخال كلمة william

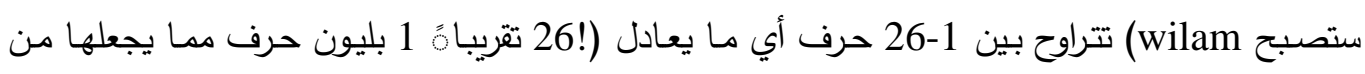
الطرق الجيدة في التثفير ). إذ يتم كتابة الكلمة في سطر والسطور التي بعده سوف تمثل جميع الحروف

\begin{tabular}{|c|c|c|c|c|}
\multicolumn{9}{|c|}{} \\
\hline w & i & l & a & m \\
\hline b & c & d & e & F \\
\hline g & h & j & k & N \\
\hline o & p & q & r & S \\
\hline t & u & v & x & Y \\
\hline z & & & & \\
\hline
\end{tabular}

2- بعدها تؤخذ الحروف من المصفوفة العلوية عمود عمود ونضعها تحت الحروف الأبجدية وكما يلي:

\begin{tabular}{|c|c|c|c|c|c|c|c|c|c|c|c|c|c|c|c|c|c|c|c|c|c|c|c|c|c|c|}
\hline alphabit & a & b & c & d & e & f & j & h & i & j & k & l & m & n & o & p & q & r & s & t & u & v & w & x & Y & z \\
\hline cipher & w & b & g & o & t & z & i & c & h & p & u & l & d & J & q & v & a & e & k & r & x & m & F & n & s & y \\
\hline
\end{tabular}

3- بعد أن حصلنا على الـ cipher يتم إدخال النص المراد تثفيره plain text (مثثلا

النص يؤخذ ما يقابل alphabet من حرف cipher لتعطي الرسالة المشفرة (ctlv). 
4- ولاسترجاع النص الأصلي من النص المشفر يتم اعتماد ذات الطريقة مع اخذ قيم alphabet بدل من الـ.

$$
\text { cipher }
$$

إن هذه الطريقة لا تتعامل فقط مع الحروف الأبجدية بل مع الأرقام مثلاً إذ يتم تعويضها بذات القيمة (مثلا الرقم 1 سيعوض عنها بالرقم 1 في النص المشفر بدون تغيير)[10]. 5- الطريقة المقترحة لإخفاء البيانات واسترجاعها:

لغرض إخفاء البيانات سنفترض إن C عبارة عن صورة ملونة التي تمثل صورة الغطاء بإبعاد

والتي تمثل بالمعادلة (1): (1)

$\mathrm{C}=\left\{\mathrm{x}_{\mathrm{ij}} \mid 0 \leq \mathrm{i}<\mathrm{M}_{\mathrm{c}}, 0 \leq \mathrm{j}<\mathrm{N}_{\mathrm{c}}, \mathrm{x}_{\mathrm{ij}} \in\{0,1, \ldots ., 255\}\right\}$

و الرسالة السرية وتمثل بالمعادلة (2)-bit تمثل:

$\mathrm{M}=\left\{\mathrm{m}_{\mathrm{i}} \mid 0 \leq \mathrm{i}<\mathrm{n}, \mathrm{m}_{\mathrm{i}} \in\{0,1\}\right\}$

ولنفترض أن n-bit للرسالة السرية سوف يتم تضمينها في k-rightmost LSB للصورة الغطاء C. سوف نتبع خطوات الخوارزمية التالية لإخفاء البيانات ( انظر الثكل 2):

1- التقاط الصورة C. - C.

2- تعيير الصورة لتصبح ضمن الإبعاد القياسية التي تم اعتمادها خلال البحث إذ يتم معالجة الصورة بإبعاد

3- تحويل الصورة إلى صورة رمادية (gray scale).

4- حساب معاملات التحويل المويجي للصورة C إذ تم اعتماد المستوى الأول أو المستوى الثاني.

5- حساب حد العتبة للمعاملات Low-high.

6- حساب عدد المواقع التي يمكن تضمين الرسالة بها لتحديد أعلى حد لعدد البت التي يمكن تضمينها.

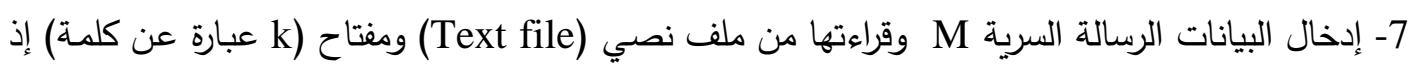

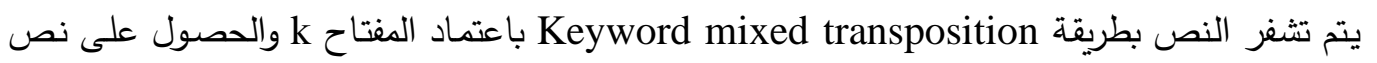
مشفر يتم تحويله من الثكل الرقمي (Decimal) إلى الثكل الثنائي (Binary).

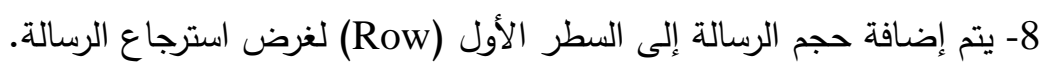

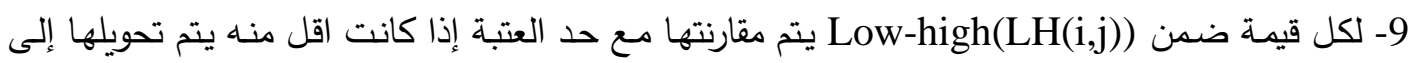
الثكل الثنائي وإبدال البت LSB بالبت من Mi للحصول على cover image، إذ أن i,j تمثل مؤشرات

$$
\text { إلى احد المعاملات للصور الرقمية. }
$$

10- إرجاع قيمة Low-high(LH(i,j)) إلى الثكل الرقمي وتكرارها إلى أن يتم إضافة جميع قيم الرسالة.

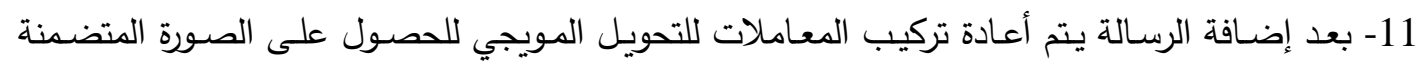
للرسالة (stego image).

ولاستعادة النص والحصول على الرسالة المشفرة نتبع الخطوات التالية كما في الثكل (3): 
1- حساب معاملات التحويل المويجي للصورة stego image التي تم الحصول عليها بعد استقبالها أو التي

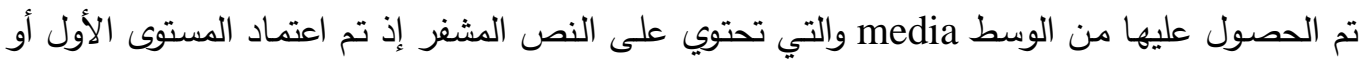

المستوى الثاني وإدخال المفتاح (k).

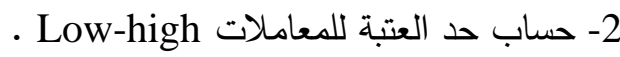

3- الحصول على حجم الرسالة من السطر الأول (Row) للمعاملات Low-high لغرض الب استرجاع الرسالة.

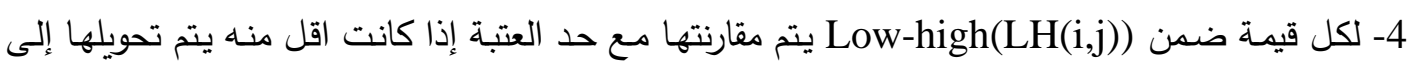

الثكل الثنائي واخذ البت LSB ووضعه في Mi تكرر العملية إلى أن يتم الوصول الحصول على جميع

البت المضمنة.

5- تحويل الـ Mi إلى الثكل الحرفي (character) وإدخاله مع المفتاح إلى طريقة الى Keyword mixed

transposition لفك شفرة النص والحصول على الرسالة السرية.
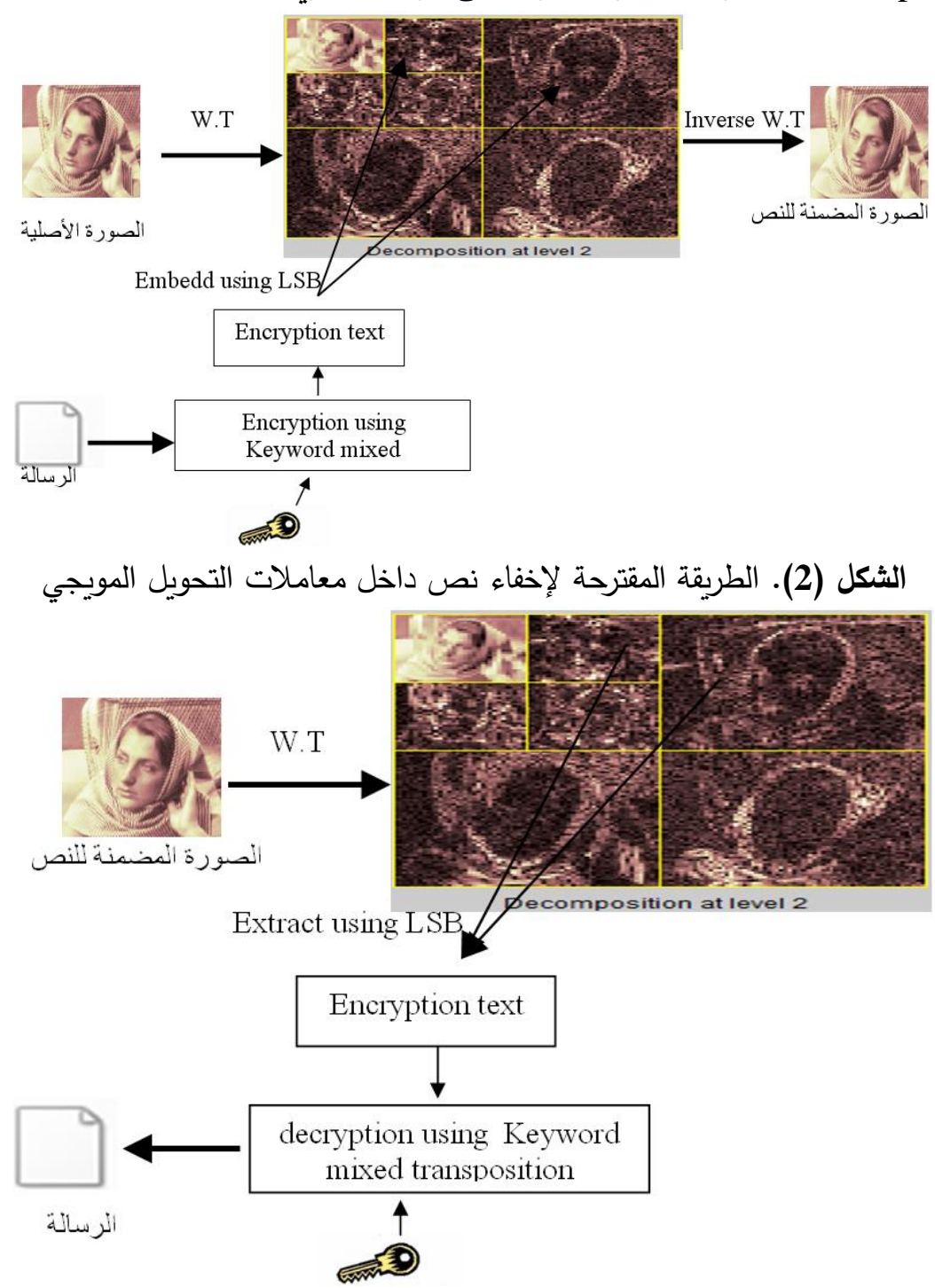

الثكل (3). الطريقة المقترحة لاسترجاع نص من داخل معاملات التحويل المويجي 
لغرض التجربة تم اختيار عدد من الصور الرقمية (ذات الامتداد و و tiff, bmp, png) ذات الحجم

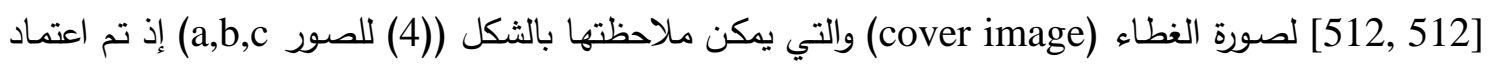

$$
\text { النص التالي (338 حرف ما يعادل } 2704 \text { بت): }
$$

A digital image processor is the heart of any image processing system. An image processor consists of a set of hardware modules that perform four basic functions: image acquisition, storage, low-level (fast) processing, and display. Typically, the image acquisition module has a TV signal as the input and converts this signal into digital form, both spatially and in amplitude.

$$
\text { واعتماد المفتاح (findyou) لاستخدامه في تثفير وفلك تثفير النص السابق وإعطاء النص التالي: }
$$

Alhihefx hnfir pzjgrddjz hd ebr brfze jw fco hnfir pzjgrddhci dodern. Ac hnfir pzjgrddjz gjcdhded jw f dre jw bfzlvfzr njlkxrd ebfe przwjzn wjkz afdhg wkcgehjcd: hnfir fgtkhdhehjc, dejzfir, xjv-xrqrx (wfde) pzjgrddhci, fcl lhdpxfo. Tophgfxxo, ebr hnfir fgtkhdhehjc njlkxr bfd f TV dhicfx fd ebr hcpke fcl gjcqrzed ebhd dhicfx hcej lhihefx wjzn, ajeb dpfehfxxo fcl hc fnpxheklr.

يتم تحويل هذا النص إلى الثكل الثنائي وتضمينه داخل معاملات التحويل المويجي حسب الطريقة المقترحة في الفقرة 5 لتكوين صورة مضمنه للنص (stego image) كما في الثكل (4) للصور de,e,f) التي من المكن

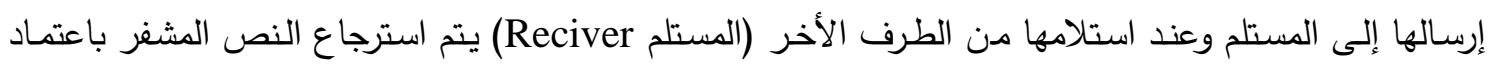
الطريقة المقترحة المذكورة أنفا ولفك تشفير النص المسترجع من الصورة المضمنة لـه ويجب استخدام نفس المفتاح للحصول على الرسالة التالية: (findyou)

Adigital image processor is the heart of any image processing system. An image processor consists of a set of hardware modules that perform four basic functions: image acquisition, storage, low-level (fast) processing, and display. Typically, the image acquisition module has a TV signal as the input and converts this signal into digital form, both spatially and in amplitude.

من خلال الجدول (1) والثكل (5) نلاحظ أن معامل الارتباط متقارب جداً (بين الصورة الأصلية والصورة المضمنة للرسالة Stego image)، كذلك يمكن ملاحظة الفرق الطفيف بين هذه الصور من خلال المقاييس

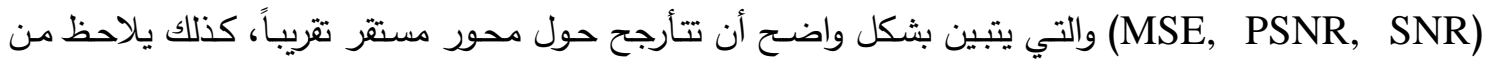

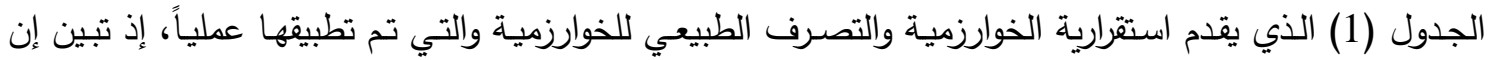
معاملات الترددات الواطئة-العالية للتحويل المويجي جيدة جداً لإخفاء البيانات من دون إن توثر على نوعية وجودة

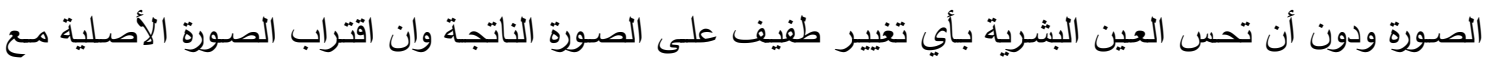
الصورة المضمنة للنص السري كان جيد مما يدعم كون الخوارزمية المعتمدة جيدة للإخفاء.

الجدول (1). قيم المعاملات (SNR, PSNR, MSE) ومعامل الارتباط

\begin{tabular}{|c|c|c|c|c||}
\hline Image & SNR & PSNR & MSE & $\begin{array}{c}\text { Correlation } \\
\text { coefficient }\end{array}$ \\
\hline \hline lena & 44.09 & 49.75 & 0.68 & 0.9998 \\
\hline barbara & 35.84 & 41.73 & 4.36 & 0.9993 \\
\hline boat & 39.13 & 44.48 & 2.31 & 0.9995 \\
\hline
\end{tabular}




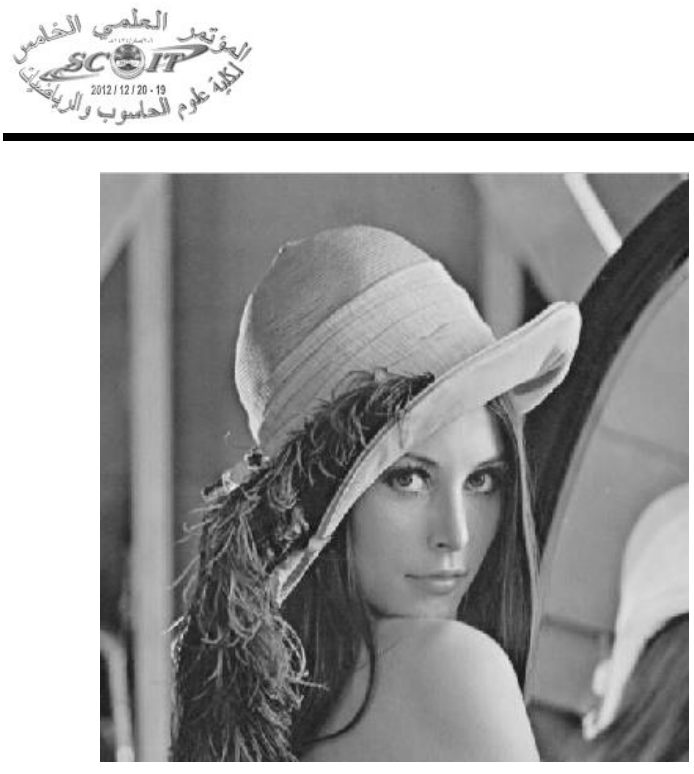

$a$

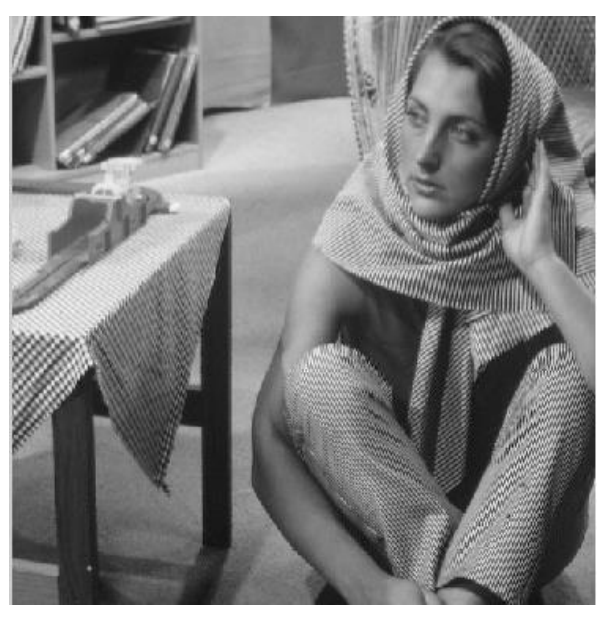

c

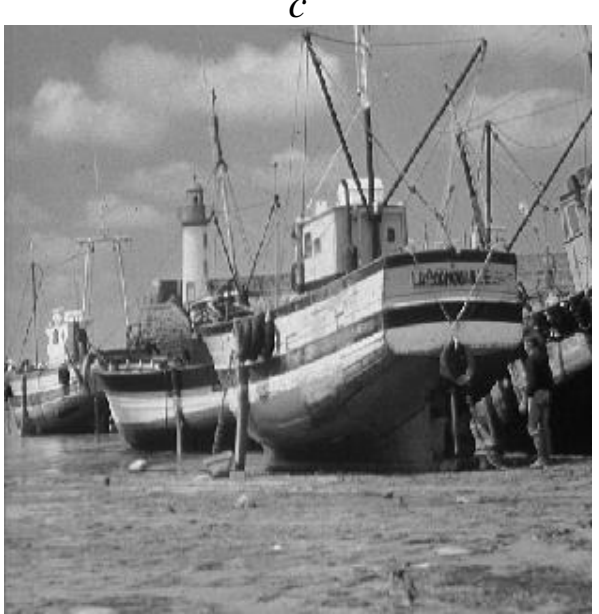

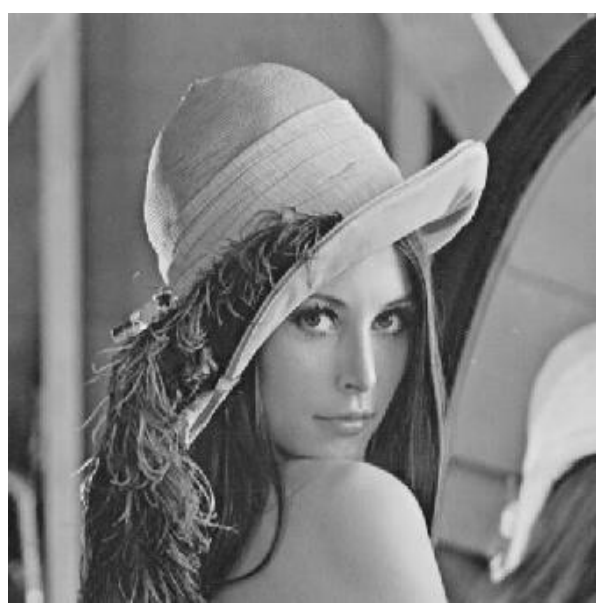

$b$

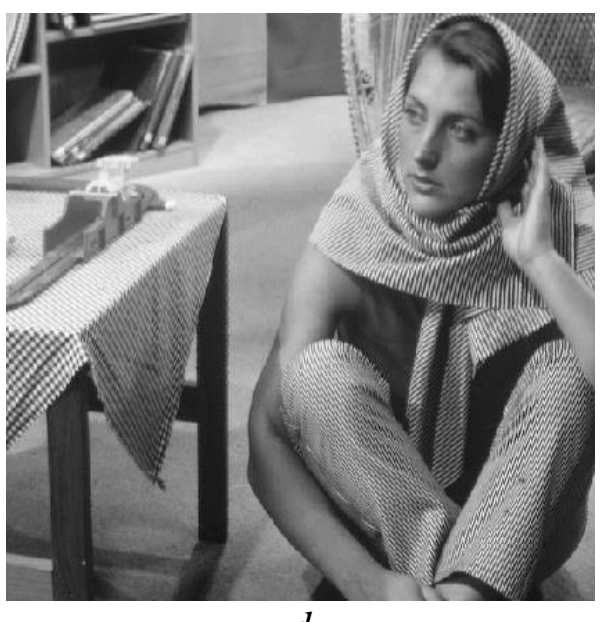

d

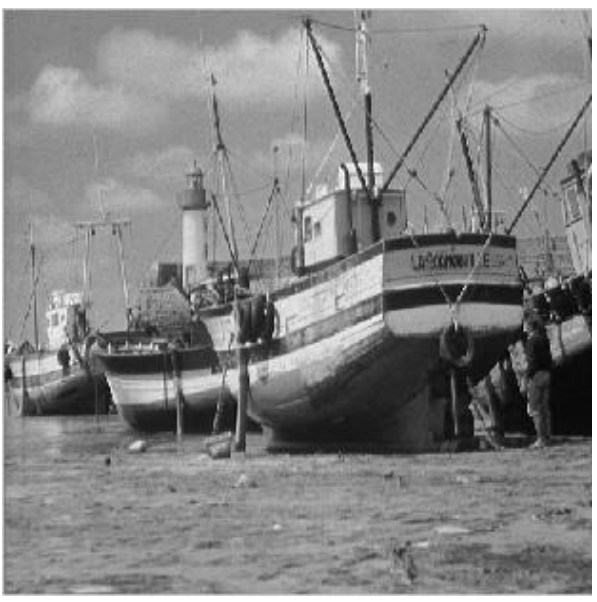

$f$

الثكل (4). يمثل بعض الصور الأصلية (a, b, c) والصور المضمنة للنص (stego image) بعد تضمين النص باعتماد الطريقة المقترحة بالبحث 


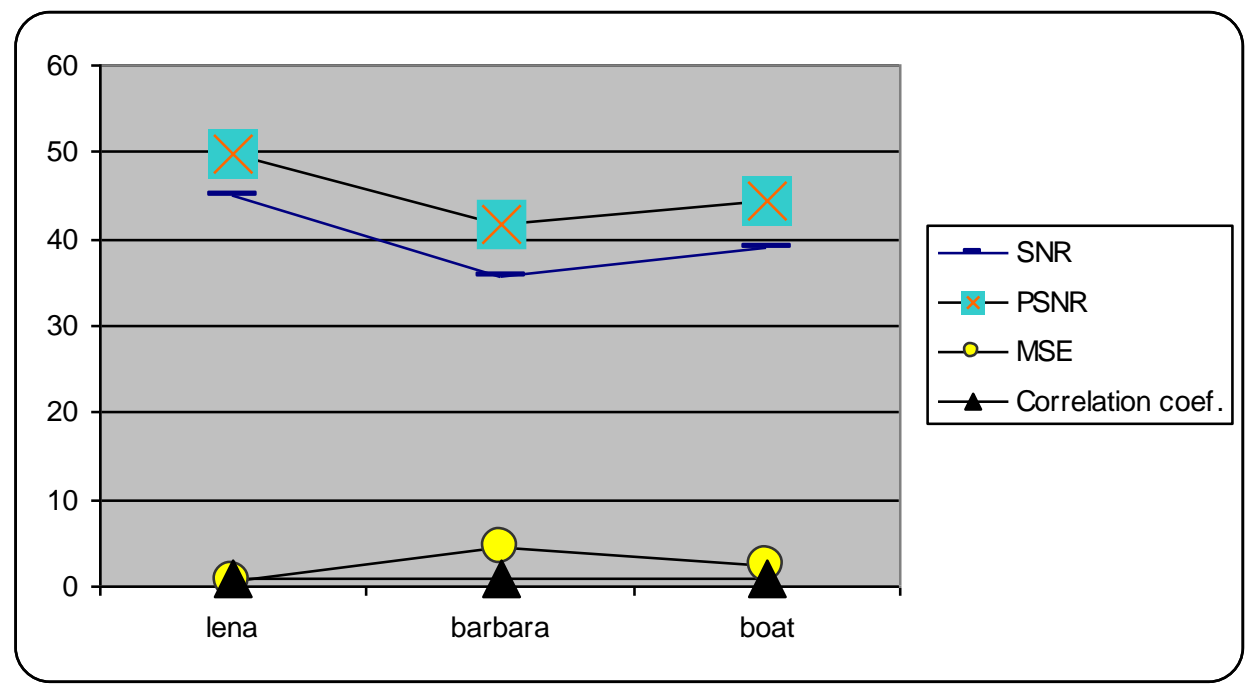

الثكل (5). رسم بياني للمعاملات MSE, PSNR, SNR ومعامل الارتباط)

7- الاستنتاجات:

إن اعتماد معاملات الترددات الواطئة- العالية للتحويل المويجي للصور الرقمية وطريقة LSB لإخفاء

البيانات توفر استقرارية عالية والتي اعتمدت من قبل الكثير من الباحثين مـع الصور الطبيعية والجويـة. وعند

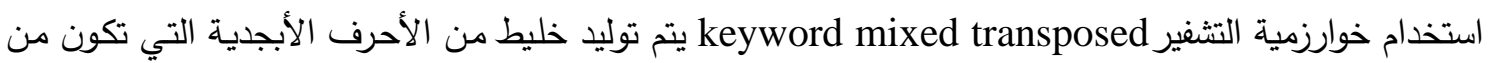
الصعب فك تشفيرها لأنه لغرض فك شفرتها يحتاج الثخص المتطفل إلى (!26) ووقت طويل جداً لفك شفرتها. إن اعتماد الخوارزمية المقترحة في البحث أعطت إمكانية عالية في تثفير وإخفاء البيانات ومقدار عالي من الإنئ الارتباط بين الصورة الأصلية والمضمنة للنص وأعلى مقاييس(PSNR, MSE, SNR) وهذا ما تم ملاحظته في الجدول

(1) والثكل (5). (1)

8 - الإعمال المستقبلية:

إمكانية اعتماد الثبكات العصبية كمعيار لقياس مدى التقارب بين الصورة الأصلية والصورة المضمنة

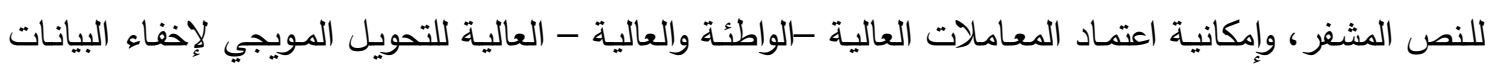
واستخدام خوارزميات تشفير أقوى وأفضل كخوارزميات DES و DSA التي تعمل على توليد أكثر من مفتاح احدها عام والآخر خاص، وإمكانية الاعتماد أكثر من بت لإخفاء البيانات. 


\section{المصادر}

[1] A.A. Abdul Latef, (2011), "Color Image Steganography Based on Discrete Wavelet and Discrete Cosine Transforms", IBN AL- HAITHAM J. FOR PURE \& APPL. SCI. VOL.24 (3).

[2] A.A. Abdelwahab, L.A. Hassan, (2008), "A discrete wavelet transform based technique for image data hiding", in: Proceedings of 25th National Radio Science Conference, Egypt.

[3] Celik, M., G. Sharma, A. M. Tekalp, and E. Saber,(2002), "Reversible data hiding", Proceedings of the International Conference on Image Processing, Rochester, NY, September.

[4] Chin-Chen Chang, (2005), "A DCT-domain System for Hiding Fractal Compressed Images", Proceedings of the 19th International Conference on Advanced Information Networking and Applications (AINA'05), Vol.2.

[5] Eman Th. Sedeek Al-obaidy,(2008), "An Algorithm for Data Hiding in Binary Images", Raf. J. of Comp. \& Math's., Vol. 5, No. 2, 8.

[6] Huang, Hui-Yu. and Shih-Hsu Chang, (2011), "Lossless Data-hiding Technique based on Wavelet Transform", MVA2011 IAPR Conference on Machine Vision Applications, June 13-15.

[7] I. Daubechies, (1992), "Ten lectures on wavelets", Philadelphis, PA: SIAM.

[8] Maity S.P. and Kundu M.K., (2004), "A Blind CDMA Image Watermarking Scheme in Wavelet Domain”, IEEE International Conference:2633 - 2336.

[9] Ni, Z., Y.Q. Shi, N. Ansari, and W. Su, (2003), "Reversible data hiding", in Proc. of 2003 Int. Symposium on Circuits and Systems, pp. II-912-915.

[10] William stallings, (1999), "Cryptography and network security: principle and practice 2nd ed", Prentice hall. upper saddle river, New Jersey 07458.

[11] Xuan G., C. Yang, Y. Zheng, Y.Q. Shi and Z. Ni, (2004), "Reversible data hiding based on wavelet spread spectrum", IEEE International workshop on multimedia signal processing (MMSP2004), Siena, Italy.

[12] Yang H., Xingming Sun and Guang Sun, (2009), "A High-Capacity Image Data Hiding Scheme Using Adaptive LSB Substitution", Radioengineering, Vol. 18, No. 4.

[13] Yong Hong Zhang, (2011), "Digital Image hiding using curvelet transform", IEEE Conference. 